\title{
NORMALIZERS OF FINITE MULTIPLICITY NESTS
}

\author{
by KENNETH R. DAVIDSON*
}

(Received 14th July 1994)

\begin{abstract}
We show that every continuous nest of bounded multiplicity is unitarily equivalent to itself in a non-trivial way. Along the way, it is shown that no finite (measurable) partition of the unit interval can separate absolutely continuous homeomorphisms.
\end{abstract}

1991 Mathematics subject classification: 47D25.

\section{Introduction}

If $\mathscr{A}$ is an operator algebra, the unitary normalizers are those unitary operators $U$ such that $U \mathscr{A} U^{*}=\mathscr{A}$. This includes, in particular, the unitary group of $\mathscr{A}$ but may often be much larger. The purpose of this note is to show that it is indeed larger in the case of continuous nest algebras of finite multiplicity. Simple examples show that infinite multiplicity nest algebras may have only trivial normalizers.

Ringrose [7] showed that every automophism $\alpha$ of a nest algebra $\mathscr{T}(\mathscr{N})$ is spatially implemented in the sense that there is an invertible operator $S$ so that $\alpha(T)=S T S^{-1}$. Because nest algebras are reflexive, $\alpha$ thus induces a dimension preserving automorphism of the nest $\mathcal{N}$ by $\theta_{\alpha}(N)=S N=\operatorname{Ran}(\alpha(N))$. It is easy to see that $\theta_{\alpha}$ is trivial precisely when $\alpha$ is inner. So $\theta$ is a natural map of $\operatorname{Aut}(\mathscr{T}(\mathscr{N}))$ into $\operatorname{Aut}(\mathscr{N})$ with kernel $\operatorname{Inn}(\mathscr{T}(\mathscr{N}))$. It is a consequence of the Similarity Theorem [2] that this map is surjective [4]. In the case of a continuous nest, the nest is parametrized by the unit interval $[0,1]$. So $\operatorname{Aut}(\mathcal{N})$ is identified with $\operatorname{Homeo}(0,1]$, the homeomorphisms of $[0,1]$ such that $h(0)=0$.

However, the unitary invariants of a nest algebra are more delicate, and often there will be no unitary operator implementing an element of $\operatorname{Aut}(\mathcal{N})$. The unitary invariants of a (parametrized) nest were obtained by Erdos [5]. These invariants consist of a spectral measure and a multiplicity function on the nest. In particular, every continuous nest may be parametrized by the unit interval $[0,1]$ as $\mathscr{N}=\left\{N_{t}\right\}$ so that the spectral measure is equivalent to Lebesque measure. The multiplicity function is then a Lebesgue measurable function of $[0,1]$ into the set $\mathbf{N} \cup\{\infty\}$. An order preserving homeomorphism $h$ of $[0,1]$ is implemented by a unitary operator exactly when $h$ and $h^{-1}$ are absolutely continuous and $h$ preserves the multiplicity function almost everywhere. So the problem may be reduced to a measure theoretic question.

*Partially supported by an NSERC grant. 
In [1], Anoussis and Katavolos analyze possible actions of the real line on a nest. When such actions are unitarily implemented and transitive, the action is unitarily equivalent to translation on $L^{2}(\mathbf{R})$ with the nest being the subspaces $L^{2}(-\infty, t)$ for real $t$. They found the example we cite below, and raise the question answered in this note about which nests have non-trivial actions implemented by unitary operators. The author wishes to thank John Orr for pointing out the Anoussis-Katavolos question which led to this paper.

We refer the reader to [3] for the necessary background on nest algebras.

Example 1. Consider a partition of $[0,1]$ into disjoint measurable sets $A_{n}$ for $n \geqq 1$ so that each $A_{n}$ meets each subinterval of $[0,1]$ in a set of positive measure. Choose a dense subset $\left\{t_{n} \mid n \geqq 1\right\}$ of $[0,1]$. Define a multiplicity function $\mu$ by setting $\mu(t)=n$ for $t$ in $A_{n}^{\prime}:=A_{n} \cap\left[0, t_{n}\right]$ for $n \geqq 1$, and equal to $\infty$ on the remainder. In order that a homeomorphism $h$ preserve the measure and multiplicity class, it must be absolutely continuous and

$$
m\left(A_{n}^{\prime} \triangle h\left(A_{n}^{\prime}\right)\right)=0 \text { for all } n \geqq 1 .
$$

Then continuity forces $h\left(\left[0, t_{n}\right]\right) \stackrel{\dot{ }}{=}\left[0, t_{n}\right]$ for all $n \geqq 1$, whence $h$ is the identity function. So this nest has no non-trivial unitary normalizers.

Surprisingly, any continuous nest of finite multiplicity has many non-trivial automorphisms which are unitarily implemented. The technique is strictly measure theoretic.

\section{Homeomorphisms of the unit interval}

Let $\operatorname{Homeo}(0,1]$ denote the homeomorphisms of $[0,1]$ such that $h(0)=0$. Likewise, let $A b s e o(0,1]$ denote the subgroup of those homeomorphisms in $\operatorname{Homeo}(0,1]$ such that $h$ and $h^{-1}$ are absolutely continuous. Say that a (Lebesgue) measurable set is essentially dense in an interval $I$ if it intersects every non-empty subinterval in a set of positive measure.

In this section, we will construct homeomorphisms of $[0,1]$ with certain properties without worrying about absolute continuity. Then in the next section, we will add the subtleties needed to obtain homeomorphisms in this restricted class.

Lemma 1.1. Let $\mathscr{S} \subset \mathbf{N}$, and let $\left\{A_{n} \mid n \in \mathscr{S}\right\}$ and $\left\{B_{n} \mid n \in \mathscr{S}\right\}$ be two partitions of $(0,1)$ into pairwise disjoint essentially dense subsets. Then there is a homeomorphism $h \in$ Homeo( 0,1$]$ such that

$$
m\left(h\left(A_{n}\right) \triangle B_{n}\right)=0 \text { for all } n \in \mathscr{S}
$$

Proof. For clarity, we first suppose that $\mathscr{S}=\{1,2\}$; so that we have two partitions $\left\{A, A^{c}\right\}$ and $\left\{B, B^{c}\right\}$ into two essentially dense subsets. Fix a sequence $\varepsilon_{n}$ tending to 0 . Then find a closed subset $A_{1} \subset A$ such that $m\left(A \backslash A_{1}\right)<\varepsilon_{1}$ and $m\left(A_{1} \cap I\right)>0$ for every interval $I$ of length $\varepsilon_{1}$. $A_{1}$ is necessarily a Cantor set because $A$ has no interior. 
Similarly, construct a set $B_{1} \subset B$ with the same properties. By a well known construction, there is an order preserving homeomorphism of $A_{1}$ onto $B_{1}$. Extend it by linearity on the components of the complement to obtain a homeomorphism $g_{1}$ of $[0,1]$.

Now on each interval $I$ of $B_{1}^{c}$, construct closed subsets $C$ of $I \cap g_{1}\left(A^{c}\right)$ and $D$ of $I \cap B^{c}$ such that

$$
m\left(I \cap g_{1}\left(A^{c}\right) \backslash C\right)<\varepsilon_{1} m(I) \text { and } m\left(I \cap B^{c} \backslash D\right)<\varepsilon_{1} m(I),
$$

and both $C$ and $D$ meet each interval of length $\varepsilon_{1}$ in a set of positive measure. As in the previous paragraph, there is an order preserving homeomorphism of $I$ which carries $C$ onto $D$. Let $h_{1}$ be the map obtained by setting it to be the identity on $B_{1}$ and patching together these homeomorphisms constructed on each interval $I$.

The composition $f_{1}=h_{1} g_{1}$ now maps a large portion of $A$ and $A^{c}$ onto a large portion of $B$ and $B^{c}$ respectively. This procedure can now be iterated. At the nth stage, a homeomorphism $f_{n}$ is constructed so that $f_{n}\left(A_{n}\right)=B_{n}$ and $f_{n}\left(C_{n}\right)=D_{n}$ where $A_{n}, B_{n}, C_{n}$ and $D_{n}$ are nested sequences of closed subsets of $A, B, A^{c}$ and $B^{c}$ respectively with

$$
\max \left\{m\left(A \backslash A_{n}\right), m\left(B \backslash B_{n}\right), m\left(A^{c} \backslash C_{n}\right), m\left(B^{c} \backslash D_{n}\right)\right\}<\varepsilon_{n},
$$

and they each meet each interval of length $\varepsilon_{n}$ in a set of positive measure. Then $f_{n+1}$ is constructed so that $f_{n+1}$ agrees with $f_{n}$ on $A_{n} \cup C_{n}$. This forces $f_{n+1}$ to map each interval of the complement to itself. As these intervals have length at most $\varepsilon_{n}$, the sequence $f_{n}$ converges uniformly to a homeomorphism $f$ with the desired properties.

If $\mathscr{S}$ is countably infinite, the procedure is similar. The iterations match up a large portion of some set $A_{k}$ with a large portion of $B_{k}$ at each stage, fixing that part that has already been aligned appropriately. A simple way to ensure that each set $A_{k}$ is adjusted infinitely often is to work with $A_{k}$ if $n \equiv 2^{k-1}\left(\bmod 2^{k}\right)$. As above the iterations converge uniformly to a homeomorphism $f$ such that $m\left(f\left(A_{k}\right) \triangle B_{k}\right)=0$ for all $k \in \mathscr{S}$.

Say that a collection of subsets $\left\{A_{\alpha} \mid \alpha \in \Omega\right\}$ essentially separates points in Homeo $(0,1]$ if

$$
m\left(h\left(A_{\alpha}\right) \triangle g\left(A_{\alpha}\right)\right)=0 \text { for all } \alpha \in \Omega
$$

implies that $h=g$ for all $g$ and $h$ in $\operatorname{Homeo}(0,1]$.

Theorem 1.2. No finite partition of $(0,1)$ essentially separates points of $\operatorname{Homeo}(0,1]$.

Proof. Suppose first that $\left\{A_{n} \mid n \in \mathscr{S}\right\}$ is a partition of $[0,1]$ into essentially dense subsets. Then the lemma above shows that there is a homeomorphism $h$ such that modulo null sets,

$$
h\left(A_{n} \cap[0,2 / 3]\right)=A_{n} \cap[0,1 / 3] \text { and } h\left(A_{n} \cap[2 / 3,1]\right)=A_{n} \cap[1 / 3,1]
$$

for all $n \in \mathscr{S}$. So this partition does not distinguish $h$ from the identity map. 
For the general case, proceed by induction on the cardinality $n$ of the partition. The result is obvious for the trivial partition when $n=1$. Suppose the result has been proven for partitions of cardinality less than $n$, and consider a partition $\left\{A_{1}, \ldots, A_{n}\right\}$ of cardinality $n$. If each $A_{i}$ is essentially dense, the argument of the previous paragraph produces a homeomorphism $h$ which is not separated from the identity by this partition. On the other hand, suppose that $A_{n}$ meets an interval $I$ in a set of measure 0 . Then by the induction hypothesis, there is a homeomorphism of $l$ which is not distinguished from the identity by $\left\{A_{i} \cap I, 1 \leqq i \leqq n-1\right\}$. Extending this to a homeomorphism $h$ of $[0,1]$ by setting it to be the identity on $I^{c}$ produces the desired homeomorphism.

\section{Absolute continuity}

Now we will work a little harder to produce a homeomorphism $h$ such that $h$ and $h^{-1}$ are absolutely continuous. Let $A b s e o(0,1]$ denote the group of such homeomorphisms.

Lemma 2.1. Let $A$ be a measurable subset of $[0,1]$. Then for each $\varepsilon>0, A$ contains $a$ Cantor set $C$ such that $m(A \backslash C)<\varepsilon$ and for each open subset $I \subset[0,1]$, either $I \cap C$ is empty or it has positive measure.

Proof. Let $C_{1}$ be any closed subset of $A$ such that $m(A \backslash C)<\varepsilon / 2$. Let $J_{1}$ be a dense open subset of $[0,1]$ of measure less than $\varepsilon / 2$, and let $C_{2}=C_{1} \backslash J_{1}$. (This step is unnecessary if $A^{c}$ is essentially dense.) So $C_{2}$ is a Cantor set. Let $\left\{I_{\alpha}\right\}$ be the collection of all open intervals such that $m\left(I \cap C_{2}\right)=0$. Let $J_{2}$ be the union of this collection. Since every union of open subsets of $[0,1]$ is the union of a countable subcollection, we see that $m\left(J_{2} \cap C_{2}\right)=0$. Let $C=C_{2} \backslash J_{2}$. This clearly has the desired properties.

Lemma 2.2. Let $S=\left\{s_{n} \mid n \geqq 1\right\}$ and $T=\left\{t_{n} \mid n \geqq 1\right\}$ be two dense subsets of $(0,1)$; and let $\varepsilon>0$ be given. Then there is a homeomorphism $h$ of $[0,1]$ such that $h(S)=T$ and $(1-\varepsilon)(a-b)<h(a)-h(b)<(1+\varepsilon)(a-b)$ for all $0 \leqq b<a \leqq 1$.

Proof. We may assume that $\varepsilon<1 / 2$, and we may add 0 and 1 to both $S$ and $T$. Fix a sequence of positive numbers $\varepsilon_{n}$ such that $\prod_{n} 1+\varepsilon_{n}<1+\varepsilon$. We will construct a bijection $h$ of $S$ onto $T$ such that

$$
(1-\varepsilon)\left(s_{i}-s_{j}\right)<h\left(s_{i}\right)-h\left(s_{j}\right)<(1+\varepsilon)\left(s_{i}-s_{j}\right)
$$

for all $s_{i}>s_{j}$ in $S$.

Begin with $h(0)=0$ and $h(1)=1$. Assume that at the $n$th stage, $h$ has been defined on a finite subset $S_{n}$ of $S$ onto a finite subset $T_{n}$ of $T$, containing $\left\{s_{1}, \ldots, s_{n}\right\}$ and $\left\{t_{1}, \ldots, t_{n}\right\}$ respectively. Moreover assume that

$$
\left(\prod_{k=1}^{n} 1-\varepsilon_{k}\right)\left(s_{i}-s_{j}\right)<h\left(s_{i}\right)-h\left(s_{j}\right)<\left(\prod_{k=1}^{n} 1+\varepsilon_{k}\right)\left(s_{i}-s_{j}\right)
$$


for all $s_{i}>s_{j}$ in $S_{n}$. If $s_{n+1} \notin S_{n}$, let $s_{i}>s_{n+1}>s_{j}$ be the nearest neighbours of $s_{n+1}$ in $S_{n}$. Pick some $h\left(s_{n+1}\right)$ in $T$ such that

$$
\left(1-\varepsilon_{n+1}\right) \frac{h\left(s_{i}\right)-h\left(s_{j}\right)}{s_{i}-s_{j}}<\frac{h\left(s_{n+1}\right)-h\left(s_{j}\right)}{s_{n+1}-s_{j}}<\left(1+\varepsilon_{n+1}\right) \frac{h\left(s_{i}\right)-h\left(s_{j}\right)}{s_{i}-s_{j}}
$$

and

$$
\left(1-\varepsilon_{n+1}\right) \frac{h\left(s_{i}\right)-h\left(s_{j}\right)}{s_{i}-s_{j}}<\frac{h\left(s_{i}\right)-h\left(s_{n+1}\right)}{s_{i}-s_{n+1}}<\left(1+\varepsilon_{n+1}\right) \frac{h\left(s_{i}\right)-h\left(s_{j}\right)}{s_{i}-s_{j}} .
$$

Then it follows immediately that

$$
\left(\prod_{k=1}^{n+1} 1-\varepsilon_{k}\right)\left(s_{n+1}-s_{j}\right)<h\left(s_{n+1}\right)-h\left(s_{j}\right)<\left(\prod_{k=1}^{n+1} 1+\varepsilon_{k}\right)\left(s_{n+1}-s_{j}\right)
$$

and

$$
\left(\prod_{k=1}^{n+1} 1-\varepsilon_{k}\right)\left(s_{i}-s_{n+1}\right)<h\left(s_{i}\right)-h\left(s_{n+1}\right)<\left(\prod_{k=1}^{n+1} 1+\varepsilon_{k}\right)\left(s_{i}-s_{n+1}\right) .
$$

Then if $s_{k}$ is another point in $S_{n}$, say without loss of generality $s_{k} \leqq s_{n+1}$, then it follows easily that

$$
\left(\prod_{k=1}^{n+1} 1-\varepsilon_{k}\right)\left(s_{n+1}-s_{k}\right)<h\left(s_{n+1}\right)-h\left(s_{k}\right)<\left(\prod_{k=1}^{n+1} 1+\varepsilon_{k}\right)\left(s_{j}-s_{k}\right) .
$$

Now let $S_{n}^{\prime}=S_{n} \cup\left\{s_{n+1}\right\}$ and $T_{n}^{\prime}=T_{n} \cup\left\{h\left(s_{n+1}\right)\right\}$. Then if $T_{n}^{\prime}$ does not contain $t_{n+1}$, proceed as above to find $h^{-1}\left(t_{n+1}\right)$ in $S$ such that for all $s_{k} \in S_{n}$, say without loss of generality $s_{k} \leqq h^{-1}\left(t_{n+1}\right)$,

$$
\left(\prod_{k=1}^{n+1} 1-\varepsilon_{k}\right)\left(h^{-1}\left(t_{n+1}\right)-s_{k}\right)<t_{n+1}-h\left(s_{j}\right)<\left(\prod_{k=1}^{n+1} 1+\varepsilon_{k}\right)\left(h^{-1}\left(t_{n+1}\right)-s_{k}\right) .
$$

Then let $S_{n+1}=S_{n}^{\prime} \cup\left\{h^{-1}\left(t_{n+1}\right)\right\}$ and $T_{n+1}=T_{n}^{\prime} \cup\left\{t_{n+1}\right\}$.

Proceeding in this way, we obtain the desired bijection. Clearly, $h$ extends by continuity to a homeomorphism $h$ of $[0,1]$ such that

$$
(1-\varepsilon)(a-b)<h(a)-h(b)<(1+\varepsilon)(a-b)
$$

for $0 \leqq b<a \leqq 1$.

Lemma 2.3. Let $A$ and $B$ be two Cantor subsets of $(0,1)$ such that every non-empty 
relatively open subset has positive measure. Then there is a homeomorphism $h \in \operatorname{Abseo}(0,1]$ such that $h(A)=B$.

Proof. Let $\alpha(t):=m([0, t] \cap A) / m(A)$, and similarly define $\beta(t)$ using the set $B$. Then the restriction of $\alpha$ to $A$ is strictly increasing except on the countable set of endpoints of the intervals in $A^{c}$. The image of this countable set is a countable dense subset $S_{\alpha}$ of $(0,1)$. Likewise, define $S_{\beta}$. Notice that a homeomorphism $k$ of $[0,1]$ which takes $S_{\alpha}$ onto $S_{\beta}$ pulls back to an order preserving homeomorphism $h$ of $A$ onto $B$. Moreover, because $\alpha$ and $\beta$ are scalar multiples of measure preserving maps, $h$ is absolutely continuous if and only if $k$ is, and likewise for $h^{-1}$ and $k^{-1}$. Moreover, if $h$ is extended to $[0,1]$ by making it linear on each component of $A^{c}$, this absolute continuity is clearly preserved.

By the previous lemma, it is possible to construct a map $k \in A b s e o(0,1]$ which takes $S_{\alpha}$ onto $S_{\beta}$. So the lemma is established.

Theorem 2.4. Let $\mathscr{S} \subset \mathbf{N}$, and let $\left\{A_{n} \mid n \in \mathscr{S}\right\}$ and $\left\{B_{n} \mid n \in \mathscr{S}\right\}$ be two partitions of $(0,1)$ into pairwise disjoint essentially dense subsets. Then there is an absolutely continuous homeomorphism $h \in \mathrm{Abseo}(0,1]$ such that

$$
m\left(h\left(A_{n}\right) \triangle B_{n}\right)=0 \text { for all } n \in \mathscr{S} .
$$

Proof. We repeat the proof of Lemma 1.1 using the previous lemmas to control the absolute continuity. Of course, a limit of absolutely continuous maps need not be absolutely continuous; but the composition of finitely many such maps is absolutely continuous. The construction has the property that it produces two partitions of the interval into countable collections of disjoint Cantor sets, say $\left\{C_{n}\right\}$ and $\left\{D_{n}\right\}$, and a homeomorphism that carries $C_{n}$ onto $D_{n}$ for all $n \geqq 1$. Moreover, this homeomorphism is the limit of homeomorphisms that are the composition of a finite number of maps which, in turn, leave the first $n$ sets $D_{i}$ fixed. Thus, the restriction of the limit to any $C_{n}$ is, in fact, arrived at in finitely many steps. By using absolutely continuous maps at each stage, we are assured that the restriction of the limit $h$ to each $C_{n}$ is an absolutely continuous homeomorphism onto $D_{n}$. As these sets have union all of $[0,1]$ except for a null set, the map $h$ is also absolutely continuous, as is its inverse.

The following corollary is immediate by the same argument as before.

Corollary 2.5. No finite partition of $(0,1)$ essentially separates points of $\mathrm{Abseo}(0,1]$.

\section{Unitary normalizers}

It is now a simple matter to obtain the desired consequences for nest algebras.

Theorem 3.1. Let $\mathcal{N}$ be a continuous nest of finite multiplicity. Then there are non-trivial unitary normalizers of $\mathcal{N}$. 
Proof. Parametrize $\mathscr{N}$ so that the spectral measure is equivalent to Lebesgue measure. Let $\mu$ be the multiplicity function of $\mathcal{N}$. Then define $A_{n}:=\mu^{-1}(n)$ for $n \leqq N$ where $N$ is the multiplicity of $\mathcal{N}$. By Corollary 2.5, there is a non-trivial homeomorphism $h \in \operatorname{Abseo}(0,1]$ which preserves this partition. By [5], there is a unitary operator $U$ which implements the induced automorphism of $\mathcal{N}$.

We immediately obtain the following corollary.

Corollary 3.2. If a continuous nest has an interval on which the multiplicity function takes only finitely many values, then it has a non-trivial unitary normalizer.

Another consequence of this argument is:

Theorem 3.3. Suppose that $\mathcal{N}$ and $\mathscr{M}$ are continuous nests such that the sets of multiplicity $n$ are essentially dense or have measure zero for each $n \in \mathbf{N} \cup\{\infty\}$. Then $\mathscr{N}$ and $\mathscr{M}$ are unitarily equivalent.

Proof. This is proven in the same manner as Theorem 3.1 using Theorem 2.4 instead of Corollary 2.5 .

This result may be considered a generalization of the Kadison-Singer Theorem [6] that any two continuous nests of multiplicity one are unitarily equivalent. Their result is an easy consequence of von Neumann's result that given any non-atomic measure with support equal to $[0,1]$, there is a reparametrization that converts this measure to Lebesgue measure. On the other hand, for arbitrary continuous nests, there still does not seem to be a clean way to describe when two of them are unitarily equivalent for some unspecified reparametrization.

For example, consider the case of multiplicity two nests. Parametrize the nest $\mathscr{N}$ so that the spectral measure is equivalent to Lebesgue measure. Then let $A_{1}$ and $A_{2}$ be the subsets of $[0,1]$ of multiplicity 1 and 2 respectively. Then let $\bar{A}_{1}$ and $\bar{A}_{2}$ denote their essential closures, i.e. $\bar{A}$ consists of all points $t$ such that $m(U \cap A)>0$ for every neighbourhood $U$ of $t$. Then $\mathcal{O}_{1}=[0,1] \backslash \bar{A}_{2}$ consists of intervals of uniform multiplicity 1 ; while $\mathcal{O}_{2}=[0,1] \backslash \bar{A}_{1}$ consists of intervals of uniform multiplicity 2 . In $[0,1] \backslash\left(\mathcal{O}_{1} \cup \mathcal{O}_{2}\right)$, both $A_{1}$ and $A_{2}$ are essentially dense. The open set $\mathcal{O}=\mathcal{O}_{1} \cup \mathcal{O}_{2}$ consists of components of multiplicities 1 and 2. So there is an associated order structure of these intervals and a map into $\{1,2\}$ that determines the multiplicity type of $\mathcal{O}$.

Now if $\mathscr{N}^{\prime}$ is another multiplicity two nest with corresponding sets $A_{1}^{\prime}, A_{2}^{\prime}, \mathcal{O}_{1}^{\prime}$ and $\mathcal{O}_{2}^{\prime}$, consider the problem of determining if it is unitarily equivalent to $\mathcal{N}$. The first difficulty is a complicated combinatorial problem to decide if the multiplicity types of $\mathscr{O}$ and $\mathcal{O}^{\prime}$ are equivalent. However, in addition there is the measure theoretic question of whether this pairing preserves the measure on the complements. The method of this note would allow the construction of an appropriate homeomorphism on the interior of the complement of $\mathcal{O}$. However, the mapping on the rest is determined by the pairing of 
intervals between $\mathcal{O}$ and $\mathcal{O}^{\prime}$. It seems unlikely to me that there is a nice simple description of when this occurs.

One could also ask about partial isometries that normalize the nest algebra in the sense that $V^{*} \mathscr{T}(\mathscr{N}) V \subset \mathscr{T}(\mathscr{N})$. For continuous nests, the situation is relatively elementary. Given any interval of a continuous nest, one may choose any vector with support equal to this interval and use it to generate a cyclic subspace for the core (the center of the diagonal). This will yield a subspace on which the nest has multiplicity one. Then given any other such subspace dominated in the order on the nest, there will exist a normalizing partial isometry in $\mathscr{T}(\mathscr{N})$ mapping the first subspace onto the second obtained using the Kadison-Singer Theorem. These partial isometries are sufficient to determine the invariant subspaces of $\mathscr{T}(\mathscr{N})$, and thus to recover it as a reflexive algebra.

On the other hand, they do not span the algebra in norm. It is not too difficult to see that, at least in the multiplicity one case, one cannot approximate finite rank elements of the nest in this way. In fact, we expect this norm closed span to be rather thin.

\section{REFERENCES}

1. M. Anoussis and A. Katavolos, Unitary actions of nests and the Weyl relations, Bull. Lond. Math. Soc. 27 (1995), 265-272.

2. K. R. Davidson, Similarity and compact perturbations of nest algebras, J. Reine. Angew. Math. 348 (1984), 286-294.

3. K. R. Davidson, Nest Algebras (Pitman Research Notes in Mathematics Series, 191, Longman Scientific and Technical Pub. Co., London, New York, 1988).

4. K. R. Davidson and B. Wagner, Automorphisms of quasitriangular algebras, J. Funct. Anal. 59 (1984), 612-627.

5. J. A. Erdos, Unitary invariants for nests, Pacific J. Math. 23 (1967), 229-256.

6. R. V. Kadison and I. M. Singer, Triangular operator algebras, Amer. J. Math. 82 (1960), 227-259.

7. J. R. Ringrose, On some algebras of operators, Proc. London Math. Soc. (3) 15 (1965), 61-83.

Pure Mathematics Department

UNIVERSITY OF WATERLOO

WATERLOO

ONTARIO N2L 3G 1

CANADA

E-mail address: krdavids@math.uwaterloo.ca 